\title{
Results and Perspectives in Solar Neutrino Detection with Borexino
}

\author{
Lino Miramonti, for the Borexino collaboration* \\ Dipartimento di Fisica dell'Università di Milano and INFN \\ Via Celoria 16, I 20133 Milano, Italy
}

\begin{abstract}
After the experimental discovery of neutrino oscillations, solar neutrino physics is about to enter the precision era. I will present the current experimental results on the fluxes of the different components of the solar neutrino spectrum in the context of the physics of neutrino oscillation and the proton fusion processes in the Sun. The perspectives of measuring neutrinos produced in the carbon-nitrogen-oxygen (CNO) fusion cycle in the Sun and the role of BOREXINO experiment will also be discussed.
\end{abstract}

Keywords: Neutrinos, Solar Standar Model, CNO-cycle,

DOI: 10.31526/ACP.NDM-2020.29

\section{INTRODUCTION}

Hydrogen burning in stars proceeds through two main thermonuclear processes, the pp-chain and the CNO-cycle [1] [2]. The ppchain dominates in low mass stars during their evolution on the main sequence. According to stellar models, more massive stars, or low mass stars that are evolved beyond the main sequence, get most of their energy through the CNO-cycle. So far there are only indirect evidence that the CNO-cycle actually operates in stars, for instance through the observation of anti-correlations in the abundances of chemical elements, carbon, nitrogen, and oxygen. Therefore, the detection of $\mathrm{CN}$ neutrinos ${ }^{1}$ would be the first direct experimental proof of the actual existence of the $\mathrm{CN}$-cycle in stars.

Although the CN-cycle is a marginal source of energy produced in the Sun (around 1\% following the results from Solar Standard Model, SSM), it is an excellent check of conditions in the solar core. We underline that SSMs are currently the most used and wellknown approach to describe the behavior of the Sun and then reproduce its characteristics, for example in terms of neutrino fluxes. There is the possibility of determining the abundance in the solar core of $\mathrm{CN}$ elements, which act as catalyzers of the cycle and this is possible because the $\mathrm{CN}$-cycle is a marginal process. The determination of the solar core abundance of $\mathrm{CN}$ elements has some relevant implications for solar and stellar physics and models. In fact, the detection of neutrinos from CNO-cycle represents an independent measurement of solar abundances that can be compared with results from spectroscopic measurements. Moreover, it breaks a degeneration between solar abundances and radiative opacities that influences neutrino fluxes from pp-chain and all robust helioseismic probes of solar interior that have prevented progress in understanding the origin of the solar abundance problem over the last 15 years. Furthermore, in the mid- and long-term timescale, it opens up the possibility of testing chemical mixing processes in the solar interior. Finally, it offers a window on the primordial composition of the solar system that has been preserved, modulo chemical changes by chemical mixing.

\section{THE PP-CHAIN}

The pp-chain starts with the fusion of two protons to form a deuteron releasing a positron and an electron neutrino [1] [2]. This reaction has a $\mathrm{Q}$ value of $420 \mathrm{keV}$ and a branching ratio of $99.6 \%$. The deuteron could be also produced, at a very low rate of $0.4 \%$, by the pep fusion (see the cyan box in Figure 1): in this case neutrinos are monochromatically emitted because of the final two-body state. Once the deuteron is created, it captures a proton giving a ${ }^{3} \mathrm{He}$ nucleus which in turn can interact in three different ways giving rise to three different branches of nuclear reactions. In the pp-I termination ${ }^{2}$, the ${ }^{3}$ He nucleus interacts with another ${ }^{3} \mathrm{He}$ nucleus forming a stable ${ }^{4} \mathrm{He}$ nucleus and releasing two protons; this happens in $85 \%$ of the cases.

* Borexino collaboration: M. Agostini, K. Altenmüller, S. Appel, V. Atroshchenko, Z. Bagdasarian, D. Basilico, G. Bellini, J. Benziger, D. Bick, G. Bonfini, D. Bravo, B. Caccianiga, F. Calaprice, A. Caminata, L. Cappelli, P. Cavalcante, F. Cavanna, A. Chepurnov, K. Choi, D. D’Angelo, S. Davini, A. Derbin, A. Di Giacinto, V. Di Marcello, X.F. Ding, A. Di Ludovico, L. Di Noto, I. Drachnev, A. Formozov, D. Franco, F. Gabriele, C. Galbiati, M. Gschwender, C. Ghiano, M. Giammarchi, A. Goretti, M. Gromov, D. Guffanti, C. Hagner, E. Hungerford, Aldo Ianni, Andrea Ianni, A. Jany, D. Jeschke, S. Kumaran, V. Kobychev, G. Korga, T. Lachenmaier, M. Laubenstein, E. Litvinovich, P. Lombardi, I. Lomskaya, L. Ludhova, G. Lukyanchenko, L. Lukyanchenko, I. Machulin, G. Manuzio, S. Marcocci, J. Maricic, J. Martyn, E. Meroni, M. Meyer, L. Miramonti, M. Misiaszek, V. Muratova, B. Neumair, M. Nieslony, L. Oberauer, V. Orekhov, F. Ortica, M. Pallavicini, L. Papp, O. Penek, L. Pietrofaccia, N. Pilipenko, A. Pocar, G. Raikov, M.T. Ranalli, G. Ranucci, A. Razeto, A. Re, M. Redchuk, A. Romani, N. Rossi, S. Rottenanger, S. Schönert, D. Semenov, M. Skorokhvatov, O. Smirnov, A. Sotnikov, Y. Suvorov, R. Tartaglia, G. Testera, J. Thurn, E. Unzhakov, A. Vishneva, R.B. Vogelaar, F. von Feilitzsch, M. Wojcik, M. Wurm, O. Zaimidoroga, S. Zavatarelli, K. Zuber, G. Zuzel

${ }^{1}$ As shown in Figure 2 neutrinos coming from the decay of ${ }^{17} \mathrm{~F}$ after proton capture on ${ }^{17} \mathrm{O}$ are negligible $(0.04 \%)$ compared to the ones emitted by the decay of ${ }^{13} \mathrm{~N}$ after proton capture on ${ }^{12} \mathrm{C}$ and the decay of ${ }^{15} \mathrm{~N}$ after proton capture on ${ }^{14} \mathrm{~N}(99.96 \%)$. The three decays constitute the CNO-cycle, while the last two decays are labeled CN-cycle. See Section 3.

${ }^{2}$ In the pp-chain there are different ways to produce ${ }^{4}$ He nuclei namely pp-I, pp-II and pp-III, see Figure 1. 


\section{pp chain}

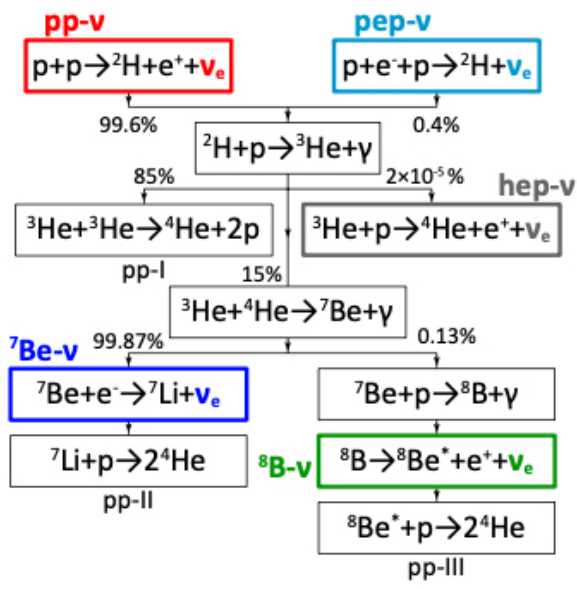

FIGURE 1: Scheme of the sequence of processes for the pp-chain according to the SSM.

In the $15 \%$ of the cases, the ${ }^{3} \mathrm{He}$ nucleus interact with a ${ }^{4} \mathrm{He}$ nucleus forming a ${ }^{7} \mathrm{Be}$ nucleus which will usually form a ${ }^{7} \mathrm{Li}$ nucleus by an electron capture, then releasing a monochomatic electron neutrino. This branch (pp-II), after a proton capture, will end with two ${ }^{4}$ He nuclei (in the $99.87 \%$ of the cases).

Once the ${ }^{7} \mathrm{Be}$ nucleus is created it could also capture a proton, which a branching ratio of $0.13 \%$, giving a ${ }^{8} \mathrm{~B}$ nucleus which undergoes a $\beta$-decay forming ${ }^{8} \mathrm{Be}$ nuclei and releasing electron neutrinos with energies up to about $16 \mathrm{MeV}$. This third pp-III termination ends with the proton capture by the ${ }^{8}$ Be nucleus then ending as two ${ }^{4} \mathrm{He}$ nuclei. With a very low branching ratio, at a level of $2 \cdot 10^{-5} \%$, instead of interacting with a ${ }^{3} \mathrm{He}$ nucleus or a ${ }^{4} \mathrm{He}$ nucleus, the ${ }^{3} \mathrm{He}$ nucleus can capture a proton forming directly a ${ }^{4} \mathrm{He}$ nucleus with emission of a positron and an electron neutrino. Figure 1 summarizes the previous sequence of processes, with their branching ratios within the pp-chain, according to SSM. In short, in the pp-chain electron neutrinos are emitted in five reactions and are labeled according to the specific reaction in which they are generated: pp- $v$, pep- $v$, Hep- $-v,{ }^{7} \mathrm{Be}-\nu$ and ${ }^{8} \mathrm{~B}-\nu$.

From the energetic point of view, pep neutrinos and ${ }^{7}$ Be neutrinos are monochromatic; pep- $v$ are emitted with $\mathrm{E}=1.44 \mathrm{MeV}$, while ${ }^{7} \mathrm{Be}-v$ present two lines, a first one at $0.384 \mathrm{MeV}$ (with a branching ratio of about $10 \%$ ) and another line at $0.862 \mathrm{MeV}$ (with a branching ratio of about $90 \%$ ).

On the contrary, the energy spectrum of pp- $v$, Hep- $v$ and ${ }^{8} \mathrm{~B}-v$ is continuous, extending up to $0.42 \mathrm{MeV}, 18.8 \mathrm{MeV}$ and $16 \mathrm{MeV}$ respectively.

In Figure 3 the fluxes of solar neutrinos from the pp-chain are thus reported, the solar neutrino energy spectrum is obtained from [4], using the updated fluxes taken from [5].

\section{THE CNO-CYCLE}

In addition to the pp chain, another set of reactions in the stars allows protons to transform into helium using ${ }^{12} \mathrm{C}$ and ${ }^{15} \mathrm{~N}$ nuclei as catalysts. These alternative thermonuclear fusion reactions constitute the so-called CNO-cycle which is composed of two subcycles: a first dominant cycle called CNO-I (or CN cycle) and a secondary one called CNO-II.

In short, the CNO-I cycle begins with the capture of a proton by a nucleus of ${ }^{12} \mathrm{C}$ which originates a nucleus of ${ }^{13} \mathrm{~N}$ which in turn rapidly decays $\beta^{+}$transforming into a nucleus of ${ }^{13} \mathrm{C}$ which, by capturing a proton, forms a nucleus of ${ }^{14} \mathrm{~N}$. After another proton capture, ${ }^{14} \mathrm{~N}$ forms a nucleus of ${ }^{15} \mathrm{O}$, which is unstable and decays $\beta^{+}$generating a nucleus of ${ }^{15} \mathrm{~N}$. In the last step of this cycle, this nucleus then captures a proton and generates a nucleus ${ }^{4} \mathrm{He}$ and one of ${ }^{12} \mathrm{C}$. The total net reaction is the fusion of four protons into a nucleus ${ }^{4} \mathrm{He}$ plus $26.731 \mathrm{MeV}$ of energy released, exactly as in the case of the pp-chain. The initial nucleus ${ }^{12} \mathrm{C}$ reappears in the last reaction and does not actively enter the reactions of the fusions but acts only as a catalyst. Within the CNO-I cycle, electronic neutrinos are emitted only and exclusively in the decays $\beta^{+}$of the nuclei ${ }^{13} \mathrm{~N}$ and ${ }^{15} \mathrm{O}$.

Once the nucleus ${ }^{15} \mathrm{~N}$ is formed, it may happen (in about $0.04 \%$ of cases) that a nucleus ${ }^{16} \mathrm{O}$ is produced instead of forming ${ }^{4} \mathrm{He}$ and ${ }^{12} \mathrm{C}$ : in this case the cycle of the subdominant $\mathrm{CNO}$-II originates. The nucleus ${ }^{16} \mathrm{O}$ then captures a proton forming a nucleus ${ }^{17} \mathrm{~F}$ which decays transforming into a nucleus ${ }^{17} \mathrm{O}$. This nucleus, capturing another proton, then forms a nucleus ${ }^{4} \mathrm{He}$ is a nucleus ${ }^{14} \mathrm{~N}$ which captures a proton becoming a nucleus ${ }^{15} \mathrm{O}$. This nucleus is unstable and undergoes decay $\beta^{+}$becoming a nucleus ${ }^{15} \mathrm{~N}$ which ends this cycle. Within the CNO-II cycle, electronic neutrinos are therefore emitted in the $\beta^{+}$decays of ${ }^{17} \mathrm{~F}$ and ${ }^{15} \mathrm{O}$ nuclei. Figure 2 summarizes the sequence of processes, with their branching relationships within the CNO-cycle according to the standard solar model.

The reaction rate that sustains the CNO-cycle depends very strongly on the star temperature and its metallicity which is strictly connected to the abundance of primordial metals in the stellar core. The full cycle can be achieved in the most central regions, where temperatures are higher than $1.33 \cdot 10^{7} \mathrm{~K}[6]$. The importance of CNO-cycle compared to the pp-chain become more relevant with the increase on the star masses being related directly with the core temperature as shown in Figure 4 where the star luminosity 


\section{CNO cycle}

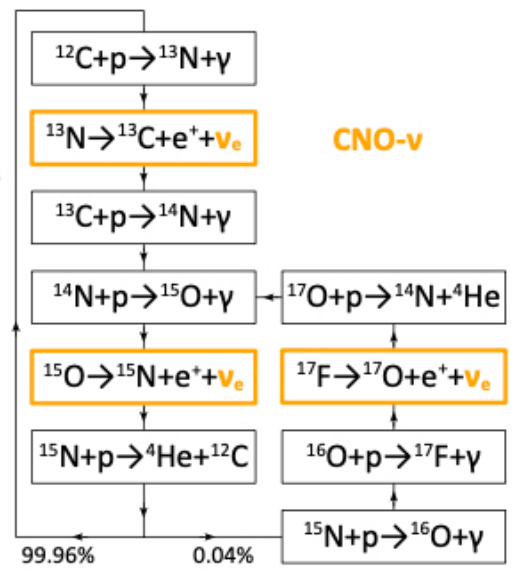

FIGURE 2: Scheme of the sequence of processes for the CNO-cycle according to the SSM.

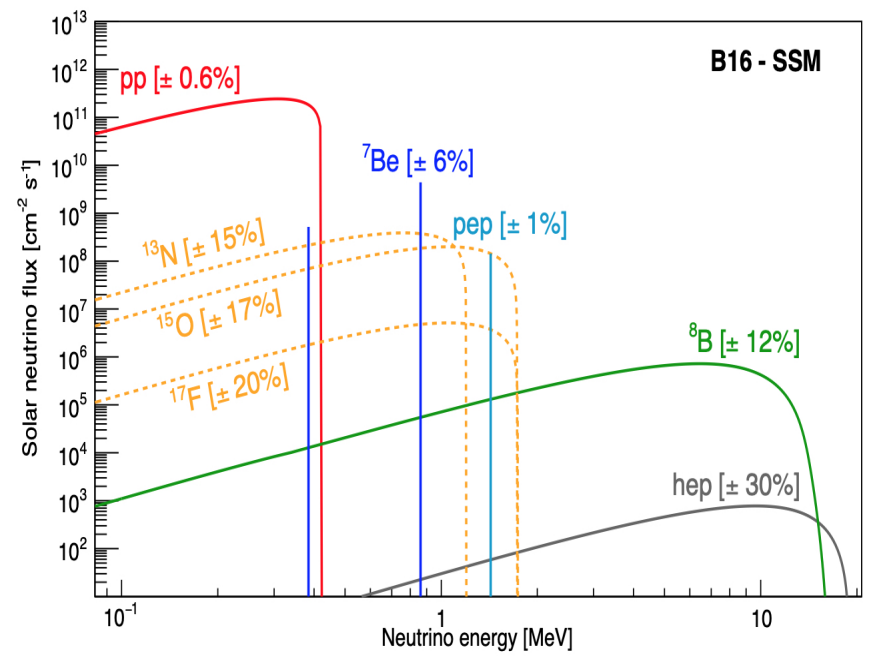

FIGURE 3: Fluxes of the solar neutrinos from the pp-chain and the CNO-cycle fusion sequences as a function of the neutrino energy. The flux (vertical scale) is given in $\mathrm{cm}^{-2} \mathrm{~s}^{-1} \mathrm{MeV}^{-1}$ units for continuum sources (pp- $v, \mathrm{Hep}-v,{ }^{8} \mathrm{~B}-v$ and CNO- $-v$ ) and in $\mathrm{cm}^{-2} \mathrm{~s}^{-1}$ units for monoenergetic sources (pep -vand ${ }^{7} \mathrm{Be}-v$ ).

is reported in log scale as a function of matter temperature for the $\mathrm{CN}$-cycle (dashed line) and pp-chain (solid line). In the figure the luminosity is normalized to the pp-chain energy production in the Sun central core $\mathrm{L} \odot$ and considering the solar metallicity and also assuming the core dynamical equilibrium. The black dot refers to the Sun core showing clearly the dominance of pp-chain at solar temperatures range, which is powered dominantly by the pp-chain reactions.

\section{THE SOLAR METALLICITY PROBLEM}

Currently, SSMs can employ two different sets of solar abundances, namely GS98 [7] for high metallization assumptions (HM) and AGSS09met [8] for low metallization hypotheses (LM). The values of the neutrino fluxes produced in the Sun and calculated in these two cases are shown in the Table 1, indicating the predictions in the case of B16-GS98 SSM and B16-AGSS09met SSM with the relative uncertainties [5]. The fourth column shows the percentage differences between the two versions.

The different metallicity values provide almost equivalent results for the neutrinos produced in the pp, pep and Hep reactions, while there is a significant difference in the case of ${ }^{7} \mathrm{Be}$ (about $8 \%$ ) and ${ }^{8} \mathrm{~B}$ (about $17 \%$ ) neutrinos. This occurs because the production of these neutrinos is more influenced by the internal temperature of the Sun and by the metallic composition of the solar matter compared to the previous neutrinos.

In the case of neutrinos produced in the $\mathrm{CNO}$ cycle, the standard solar models provide an even greater dependence on both temperature and abundance of ${ }^{12} \mathrm{C}$, and more generally on solar metallicity. Consequently, the flux values of these neutrinos obtained from the two sets of metallicity differ by approximately $26.6 \%$ for ${ }^{13} \mathrm{~N}-v, 29.8 \%$ for ${ }^{15} \mathrm{O}-v$ and up to $38.6 \%$ for ${ }^{17} \mathrm{~F}-v$, for an average value of about $28 \%$. Thanks to this great difference, greater than the uncertainties on the values themselves, the neutrino 


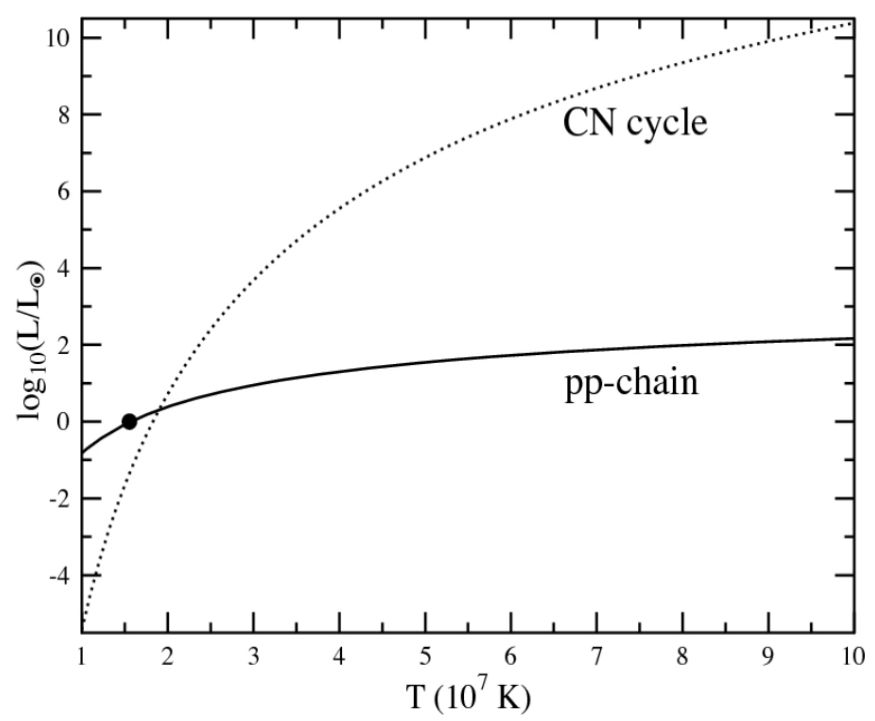

FIGURE 4: Star luminosity in log scale as a function of temperature for the pp-chain and CN-cycle.

flux could be used as a way to test the goodness of the different solar models by directly solving the relative controversy between $\mathrm{HM}$ and LM scenarios.

\begin{tabular}{c|c|c|c}
\hline Solar $v^{\prime}$ s & Flux B16-GS98 (HZ) & Flux B16-AGSS09met (LZ) & $\%$ diff. \\
\hline pp & $5.98(1.0 \pm 0.006) \cdot 10^{10}$ & $6.03(1.0 \pm 0.005) \cdot 10^{10}$ & 0.83 \\
${ }^{7}$ Be & $4.93(1.0 \pm 0.06) \cdot 10^{9}$ & $4.50(1.0 \pm 0.06) \cdot 10^{10}$ & 8.7 \\
pep & $1.44(1.0 \pm 0.01) \cdot 10^{8}$ & $1.46(1.0 \pm 0.009) \cdot 10^{9}$ & 1.4 \\
${ }^{8} B$ & $5.45(1.0 \pm 0.12) \cdot 10^{6}$ & $4.50(1.0 \pm 0.12) \cdot 10^{6}$ & 17.4 \\
Hep & $7.98(1.0 \pm 0.30) \cdot 10^{3}$ & $8.25(1.0 \pm 0.12) \cdot 10^{3}$ & 3.4 \\
\hline${ }^{13} \mathrm{~N}$ & $2.78(1.0 \pm 0.15) \cdot 10^{8}$ & $2.04(1.0 \pm 0.14) \cdot 10^{8}$ & 26.6 \\
${ }^{15} \mathrm{O}$ & $2.05(1.0 \pm 0.17) \cdot 10^{8}$ & $1.44(1.0 \pm 0.16) \cdot 10^{8}$ & 29.8 \\
${ }^{17} \mathrm{~F}$ & $5.29(1.0 \pm 0.20) \cdot 10^{6}$ & $3.26(1.0 \pm 0.18) \cdot 10^{6}$ & 38.6 \\
All CNO & $4.88(1.0 \pm 0.16) \cdot 10^{8}$ & $3.51(1.0 \pm 0.15) \cdot 10^{8}$ & 28.1 \\
\hline
\end{tabular}

TABLE 1: Predicted neutrino fluxes for the high metallicity case (second column) and low metallicity case (third column) of the Solar standard Model. Column fourth reports the differences of the two cases in percentage. Fluxes are given in $\mathrm{cm}^{-2} \mathrm{~s}^{-1}$, see text.

\section{THE BOREXINO DETECTOR}

The detector of Borexino experiment is housed in the Hall C of the Laboratori Nazionali del Grans Sasso (LNGS), which can be reached by a highway tunnel under the Gran Sasso massif in central Italy and is protected by $\sim 1.4 \mathrm{~km}$ of rock, equivalent to 3800 $\mathrm{m}$ of water. This shield strongly suppresses the cosmic radiation so that flux of cosmic muons that reaches the underground rooms is $\sim 1.2 \mu \mathrm{m}^{-2} h^{-1}$. Figure 5 shows a 3D sketch of the detector [9] .

The external structure of the detector is the Stainless Steel Sphere (SSS), with a diameter of $13.7 \mathrm{~m}$, that contains liquid scintillator and buffers. The active mass of Borexino experiment is composed by 278 tons of pseudocumene which is doped with $1.5 \mathrm{~g} / 1$ of PPO in order to enhance the scintillator properties and it is contained in a $8.5 \mathrm{~m}$ in diameter nylon Inner Vessel (its thickness of $125 \mu \mathrm{m}$ ) surrounded by two concentric pseudocumene buffers doped with a light quencher (DMP). In turn, SSS is enclosed in a Water Tank, with a shape of a dome, containing 2100 tons of ultra-pure water acting as an additional shield.

When a physical event occurs, a light is produced by this organic liquid scintillator and detected by 22128 " PhotoMultiplier Tubes (PMTs) distributed on the inner surface of SSS. Into the Water Tank, there are additional 208 8" PMTs able to detect the Čerenkov light radiated by cosmic muons. 


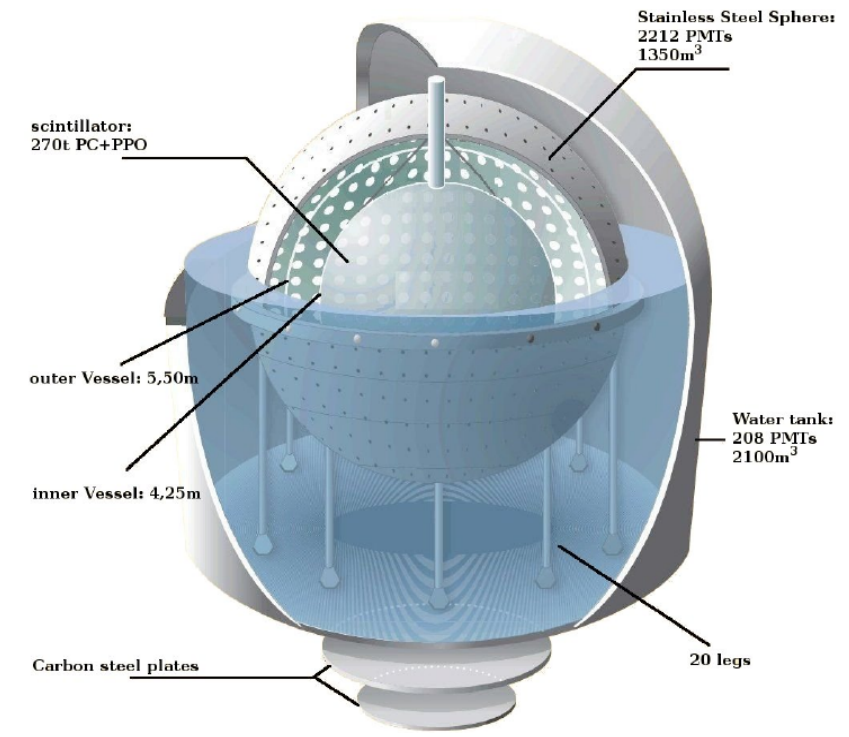

FIGURE 5: 3D sketch of the Borexino detector.

The light yield of the scintillator is $\sim 10^{4}$ photons per $\mathrm{MeV}$, corresponding to $\sim 500$ detected photoelectrons per $\mathrm{MeV}$.

The fast time response of the scintillator $(\sim 3 \mathrm{~ns})$ allows the reconstruction of the events position by means of a time-of-flight technique; the precision in position is within $\sim 13 \mathrm{~cm}$. Thanks to this accurate position reconstruction it is possible to define a "fiducial volume" ranging between 75 tons and 150 tons, depending on the analysis type.

The principle of neutrino detection is based on elastic scattering on electrons in the target material: $v_{x}+e^{-} \rightarrow v_{x}+e^{-}$.

The light produced by the scattered electron is emitted isotropically; therefore, it is impossible to distinguish light from the signal from that produced by $\beta$ and $\gamma$ rays emitted by radioactive isotopes present inside the detector. In order to reach a signal to noise ratio of the order of one, the ${ }^{238} \mathrm{U}$ and ${ }^{232} \mathrm{Th}$ content must be reduced to the level of $10^{-16} \mathrm{~g}(\mathrm{U}) / \mathrm{g}$ corresponding to about one count per day (cpd) in 100 tons.

In order to reduce the amount radioisotopes inside the scintillator, several and different techniques have been applied such as distillation, water extraction and nitrogen stripping [10].

Thanks to a purification campaign, which lasted more than two years, the Borexino Collaboration was able to reach unprecedented levels of scintillator radiopurity: the concentrations of ${ }^{238} \mathrm{U}$ and ${ }^{232} \mathrm{Th}$ are $<9.4 \cdot 10^{-20} \mathrm{~g} / \mathrm{g}$ and $<5.7 \cdot 10^{-19} \mathrm{~g} / \mathrm{g}(95 \% \mathrm{C} . \mathrm{L}$.), respectively.

\section{BOREXINO RESULTS}

Thanks to the very high levels of radiopurity indicated above, the Borexino detector allowed to detect almost all solar $v^{\prime} \mathrm{s}$ emitted within the pp-chain, with a very high energy resolution. The whole dataset was divided into two regions: a low energy region (from $190 \mathrm{keV}$ to about $2.9 \mathrm{MeV}$ ), where neutrinos from pp, pep and ${ }^{7} \mathrm{Be}$ reactions were analyzed, and a high energy region (from about $3 \mathrm{MeV}$ to about $16 \mathrm{MeV}$ ), dedicated to the study of Hep- $v^{\prime} \mathrm{s}$ and ${ }^{8} \mathrm{~B}-v^{\prime} \mathrm{s}$.

Table 2 (adapted from [12]) shows the Borexino results on solar neutrinos; in the third column the non-oscillated fluxes assume the MSW-LMA oscillation parameters [11] are reported. The result for pep- $v^{\prime}$ s depends on whether we assume HZ or LZ metallicity for constraining the CNO contribution.

The ${ }^{7} \mathrm{Be}-v$ signal has been measured with a very high precision of about $2.7 \%$, to be compared with the value of about $8 \%$ for ${ }^{8} \mathrm{~B}-v$ while for the pp- $v$, pep- $v$ the precision in $10 \%$ and $16 \%$ respectively. The only missing measurement within the pp-chain is the component due to Hep- $v$. In this case, only an upper limit of $<2.2 \cdot 10^{5}$ (90\% C.L.) on the neutrino flux has been put.

An upper limit has also been put for neutrinos coming from the CNO-cycle. The obtained value of $<7.9 \cdot 10^{8} \mathrm{~cm}^{-2} \mathrm{~s}^{-1}(95 \%$ C.L.) has to be compared with $4.88(1.0 \pm 0.11) \cdot 10^{8} \mathrm{~cm}^{-2} \mathrm{~s}^{-1}$ from SSM in the HZ case and $3.51(1.0 \pm 0.10) \cdot 10^{8} \mathrm{~cm}^{-2} \mathrm{~s}^{-1}$ for the LM case.

\section{TOWARD CNO- $v$ DETECTION}

If we compare the measured upper limit of $<8.1 \mathrm{cpt} / 100 \mathrm{t}(95 \%$ C.L) with what SSM predicts for HZ and LZ cases (4.91 \pm $0.52 \mathrm{cpd} / 100 \mathrm{t}$ and $3.52 \pm 0.37 \mathrm{cpd} / 100 \mathrm{t}$, respectively), the CNO- $v$ detection is not so far away to reached. Nevertheless, radioactive backgrounds are actually the most serious challenger to measure $\mathrm{CNO}$ neutrinos. 


\begin{tabular}{lll}
\hline Solar $v$ & Rate $(\mathrm{cpt} / 100 \mathrm{t})$ & Flux $\left(\mathrm{cm}^{-2} \mathrm{~s}^{-1}\right)$ \\
\hline$p p$ & $134 \pm 10_{-10}^{+6}$ & $\left(6.1 \pm 0.5_{-0.5}^{+0.3}\right) \cdot 10^{10}$ \\
${ }^{7} \mathrm{Be}$ & $48.3 \pm 1.1_{-0.7}^{+0.4}$ & $\left(4.99 \pm 0.11_{-0.08}^{+0.06}\right) \cdot 10^{9}$ \\
$\operatorname{pep}(\mathrm{HZ})$ & $2.43 \pm 0.36_{-0.22}^{+0.15}$ & $\left(1.27 \pm 0.19_{-0.12}^{+0.08}\right) \cdot 10^{8}$ \\
$\operatorname{pep}(\mathrm{LZ})$ & $2.65 \pm 0.36_{-0.24}^{+0.15}$ & $\left(1.39 \pm 0.19_{-0.13}^{+0.08}\right) \cdot 10^{8}$ \\
${ }^{8} \mathrm{~B}$ & $0.223_{-0.016-0.006}^{+0.015+0.006}$ & $\left(2.57_{-0.19-0.07}^{+0.17}\right) \cdot 10^{6}$ \\
Hep & $<0.002(90 \% C . L)$. & $<2.2 \cdot 10^{5}(90 \%$ C.L. $)$ \\
CNO & $<8.1(95 \%$ C.L. $)$ & $<7.9 \cdot 10^{8}(95 \%$ C.L. $)$ \\
\hline
\end{tabular}

TABLE 2: Borexino results on solar neutrinos rates and fluxes. The third column shows the non-oscillated fluxes assume the MSW-LMA oscillation parameters [11]. (table adapted from [12])

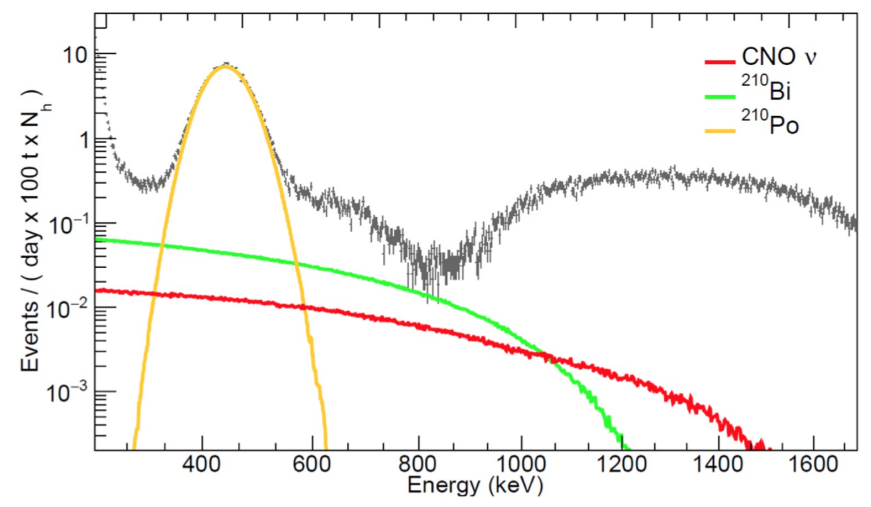

FIGURE 6: The ${ }^{210} \mathrm{Bi}$ beta-spectrum (in green) with the one of electrons recoiling off CNO- $v$ (in red) along the ${ }^{210}$ Po alpha-spectrum (in yellow).

The measurement of the CNO- $v$ flux is of paramount importance in order to understand the evolution of stars and to address the controversy on the solar metallicity. Since 2015, the Borexino Collaboration has undertaken significant efforts to achieve an independent measurement of the background which affect the CNO- $v$ detection.

This measurement is very challenging because of the low rate expected in the Borexino detector, the lack of prominent spectral features and the presence of ${ }^{210} \mathrm{Bi}(\beta$ emitter) which decaying in the liquid scintillator creates events with an energy distribution that is very close to that of $\mathrm{CNO}-v$ interactions.

The ${ }^{210} \mathrm{Bi}$ is coming from the noble gas ${ }^{222} \mathrm{Rn}$ and for this reason, it is found in the air and practically on all surfaces. It is sustained by the long-lived ( $\tau=22.3$ y) $\beta$ emitter ${ }^{210} \mathrm{~Pb}$, and is followed by the relatively long-lived $\tau=138 \mathrm{~d}$ ) $\alpha$ emitter ${ }^{210} \mathrm{Po}$.

As shown in Figure 6, the ${ }^{210} \mathrm{Bi} \beta$ spectrum (in green) is quasi-degenerate with that of electrons recoiling off CNO- $v$ (in red). Thus, to extract the CNO- $v$ signal, a very accurate measure of the ${ }^{210} \mathrm{Bi}$ background is required. A possible way to fix this contribution is to measure the supported ${ }^{210}$ Po component after the fraction which is out of equilibrium has decayed away.

A precise measurement of the steady-state component is quite difficult because of to the background fluctuations caused by mixing of the scintillator due to convective motions inside the detector. To prevent/suppress these fluctuation movements, the entire Borexino detector was thermally isolated from the air of the experimental room. As shown in Figure 7, the Collaboration has managed to thermally stabilize the detector and hopes to limit the contribution of ${ }^{210} \mathrm{Bi}$ in order to allow a measurement of $\mathrm{CNO}-v$ in the next months.

\section{ACKNOWLEDGEMENTS}

The Borexino program is made possible by funding from INFN (Italy), NSF (USA), BMBF, DFG (OB168/2-1, WU742/4-1, ZU123/18-1), HGF, and MPG (Germany), RFBR (Grants 16-02-01026 A, 15-02-02117 A, 16-29-13014 ofim, 17-02-00305 A), RSF (Grant 17-02-01009) (Russia), and NCN (Grant UMO 2013/10/E/ST2/00180) (Poland). 


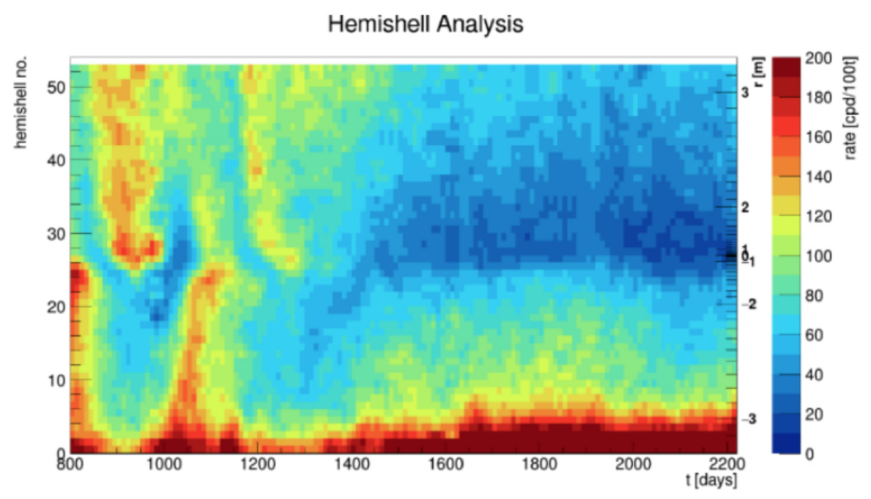

FIGURE 7: Layering of the ${ }^{210}$ Po activity inside the fiducial volume.

\section{References}

[1] V.Antonelli et al. Hindawi Publishing Corporation Advances in High Energy Physics Volume 2013, Article ID 351926, 34 pages. http://dx.doi.org/10.1155/2013/351926

[2] L.Miramonti and F .Reseghetti, "Solar neutrino physics: historical evolution, present status and perspectives", Rivista del Nuovo Cimento, vol. 25, no. 7, pp. 1-128, 2002.

[3] E. Ma and G. Rajasekaran, Phys. Rev. D64, 113012 (2001).

[4] url: http://www.sns.ias.edu/ jnb/

[5] N. Vinyoles et al. Astrophys. J. 835.2 (2017), p. 202.

[6] W. C. Haxton, R. G. Hamish Robertson, and Aldo M. Serenelli. Ann. Rev. Astron. Astrophys. 51 (2013), p. 21.

[7] N. Grevesse and A. J. Sauval. Space Sci. Rev. 85 (1998), p. 161.

[8] M. Asplund et al. Annual Review of Astronomy and Astrophysics 47 (2009).

[9] G.Alimontiet al., NIM A 2009, V.600, P.568.

[10] G.Alimonti et al., NIM A 2009, V.609, P.58.

[11] I. Esteban et al. Journal of High Energy Physics 01, (2017)

[12] Borexino Collaboration Nature 562, 505 (2018) 